\title{
EXISTENCE OF POSITIVE SOLUTION FOR A SINGULAR SYSTEM INVOLVING GENERAL QUASILINEAR OPERATORS
}

\section{Francisco Julio S.A. CorrêA, Amanda Suellen S.C. Leão and GIOVANY M. FigUEIREDO}

Abstract. In this paper we study a result of existence of positive solution the following class of singular system:

$$
\left\{\begin{array}{l}
-\operatorname{div}\left(a_{1}\left(|\nabla u|^{p_{1}}\right)|\nabla u|^{p_{1}-2} \nabla u\right)=h_{1}(x) v^{-\gamma_{1}}+k_{1}(x) v^{\alpha_{1}} \text { in } \Omega \\
-\operatorname{div}\left(a_{2}\left(|\nabla v|^{p_{2}}\right)|\nabla v|^{p_{2}-2} \nabla v\right)=h_{2}(x) u^{-\gamma_{2}}+k_{2}(x) u^{\alpha_{2}} \text { in } \Omega, \\
u, v>0 \text { in } \Omega \\
u=v=0 \text { on } \partial \Omega
\end{array}\right.
$$

where $\Omega$ is a bounded smooth domain in $\mathbb{R}^{N}$ with $N \geqslant 3,2 \leqslant p_{1}, p_{2}<N$. For $i=1,2$, $\alpha_{i}, \gamma_{i} \in\left(0, p_{i}-1\right)$ and $h_{i}$ and $k_{i}$ are continuous functions. The hypotheses on the functions $a_{i}: \mathbb{R}^{+} \rightarrow \mathbb{R}^{+}$allow to show that $(P)$ includes a large class of systems. We use topological arguments to show the main result.

Mathematics subject classification (2010): 35J60, 35J92.

Keywords and phrases: p\&q-Laplacian, singular problem, global continuum.

\section{REFERENCES}

[1] C.O. Alves, F.J.S.A. Corrêa, On the existence of positive solution for a class of singular systems involving quasilinear operators, Appl. Math. Comput., 185 (2007), 727-736.

[2] C.O. Alves, F.J.S.A. Corrêa, J. V. A. Gonçalves, Existence of solutions for some classes of singular Hamiltonian systems, Advanced Nonlinear Studies, 5 (2005), 265-278.

[3] R.P. Agarwal, D. O'Regan, Existence theory for single and multiple solutions to singular positive boundary value problems, J. Differ. Equations, 175 (2001), 393-414.

[4] H. Brezis, Functional Analysis, Sobolev Spaces and Partial Differential Equations, Springer, Paris, 2011.

[5] M.G. Crandall, P.H. Rabinowitz, L. Tartar, On a Dirichlet problem with singular nonlinearity, Comm. Partial Diff. Equat., 2 (1977), 193-222.

[6] M. del Pino, A priori estimates applications to existence-nonexistence for a semilinear elliptic system, Indiana Univ. Math. J., 43 (1994), 77-129.

[7] E. Di Benedetto, Local regularity of weak solutions of degenerate elliptic equations, Nonlinear Analysis, 7 (1983), 827-850.

[8] G.M. Figueiredo, Existence of positive solutions for a class of $p \& q$ elliptic problems with critical growth on $\mathbb{R}^{N}$, J. Math. Anal. Appl., 378 (2011), 507-518.

[9] G.M. Figueiredo, Existence and multiplicity of solutions for a class of $p \& q$ elliptic problems with critical exponent, To appear in Mathematische Nachrichten.

[10] A. Gierer and H. Meinhardt, A theory of biological pattern formation, Kybernetik, 12 (1972), 30-39.

[11] O. Kavian, Inegalité de Hardy-Sobolev et applications, Thése de Doctorate de 3 eme cycle, Université de Paris VI (1978).

[12] C.D. Luning, W.L. Perry, Positive solutions of negative exponent generalized Emden-Fowler boundary value problem, SIAM J. Math. Anal., 12 (1981), 874-879. 
[13] P.H. Rabinowitz, Some global results for nonlinear eigenvalue problems, J. Funct. Anal., 7 (1971), 487-513.

[14] S. Sakaguchi, Concavity properties of solutions to some degerate quasilinear elliptic Dirichlet Problems. Ann. Scuola Normale Sup. di Pisa, Série 4, 14 (1987), 403-421.

[15] J. Serrin, Local behavior of solutions of quasi-linear elliptic equations, Acta Math., 111 (1964), 247 302.

[16] C.A. Stuart, Existence and approximations of solutions of nonlinear elliptic equations, Math. Z., 147 (1976), 53-63.

[17] S. Taliaferro, A nonlinear singular boundary value problem, Nonlinear Analysis, (1979), 897-904.

[18] P. Tolksdorff, Regularity for a more general class of quasilinear elliptic equations, J. Differ. Equations, 51 (1984), 126-150.

[19] Z. Zhang, On a Dirichlet problem with a singular nonlinearity, J. Math. Anal. Appl., 194 (1995), $103-113$.

[20] Z. Zhang, Positive solutions of Lane-Emden systems with negative exponents: Existence, boundary behavior and uniqueness, Nonlinear Analysis, 74 (2011), 5544-5553. 